\title{
The Establishment of Partnerships to Create Virtual Organizations: A Multiagent Approach
}

\author{
M. A. Hochuli Shmeil, E. Oliveira \\ Faculdade de Engenharia da Universidade do Porto \\ Rua dos Bragas, 4099 Porto Codex, Portugal \\ Tel+351-2-2004527 E-mail: \{shmeil, eco\}@fe.up.pt
}

\begin{abstract}
Virtual Organizations are aggregations of independent organizations or individuals aiming to contribute to a common goal. Two basic steps are needed to assemble a virtual organization: (i) a business process partitioning, and (ii) a partners' selection process. Broadly speaking, in this paper we present a computational approach to model organizations based on Distributed Artificial Intelligence - Multiagent Systems (DAI-MAS) as well as Symbolic Learning (SL) paradigms. Each organization, which is seen as an agent, is provided with the needed observation, planning, coordination, execution, communication and learning capabilities to perform its social roles. In particular, we present a specific inter-organization relationship: the selection process that leads to the automatic establishment of contracts between organizations. This selection process is composed of a bid evaluation phase followed by a negotiation phase as a mean for agents conflicts resolution. Through negotiation interactions, a set of offer and counter-offer values which are seen as instances (positives and negatives) are supplied for further analysis in order to support the learning activities. The contribution of our work lies, not only, on the computational model proposed for the society of organizations, but also in some extent, on the learning methodologies applied to the established partnerships, in particular, and to the community, in general.
\end{abstract}

\section{Keywords}

Multiagent system, Organization Modeling, Application.

\section{INTRODUCTION}

The goods and services market is composed of two types of economic agents, the organizations and the individuals, related by their interactions. They can, dynamically, assume productive, distributive and consumptive basic roles. The interactions play a decisive part in the behavior of the organizations and individuals, and are used to: (i) decide what and how to produce, (ii) indicate where and how to distribute the production and (iii) determine how to promote and validate the products. In our scenario proposal (Figure 1), the involved organizations are conceived as goods and services producers, consumers or distributors (suppliers), depending on the role they play in each interaction. In this scenario individuals are consumers. To model this domain, an under developing computational system called ARTOR (ARTificial ORganizations) uses Distributed Artificial Intelligence-Multi Agent System (DAI-MAS) and Symbolic Learning 
(SL). Its goal is, to create, maintain and provide an adequate setting where organizations and their interactions are simulated (Figure 2). In our first approach we are focusing the hierarchical form as an organizational model.

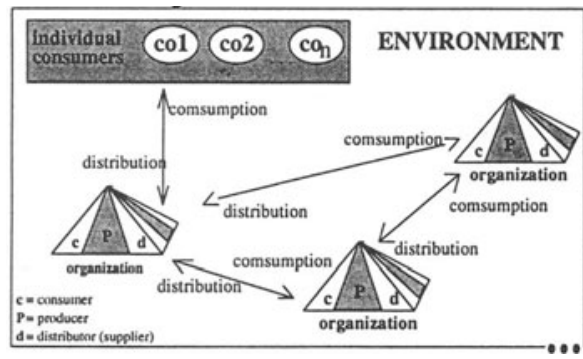

Figure 1 - The scenario proposal.

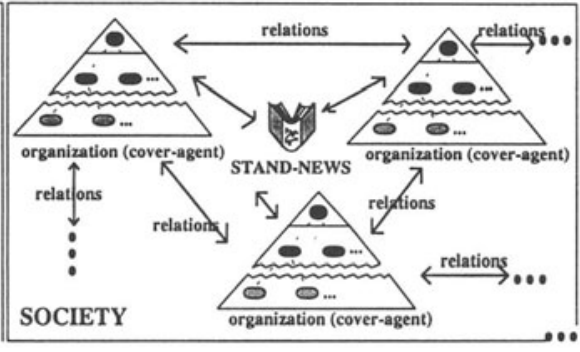

Figure 2 - The Multiagent society.

In this setting each organization acts as a cognitive agent with the capability of performing any of the following economic roles: production, distribution (supplying), and consumption. These roles or behaviors are dynamically enabled/disabled by the established interactions. The agents that map such organizations are referred as cover-agents (Figure 3). Cover-agents are semi-autonomous agents that need to establish and maintain interactions in order to "survive". Cover-agents are implemented as a set of finer grain agents. The inter-cover-agent communication can be of three types: (i) point to point, when a cover-agent addresses its message or observation to another cover-agent, (ii) point to multi-point, when a cover-agent addresses its message or observation to more than one cover-agent, and (iii) newspaper-like, when a cover-agent wishes to make public some data. The latter is built using a blackboard [1] called stand-news, where the data is posted and kept for public consultation. From the society point of view, the cover-agents are distributed agents, that act in conformity with their cognitive capabilities ${ }^{1}$.

\section{THE INNER OF A COVER-AGENT}

The adopted cover-agent architecture (related to our actual focus - the hierarchical organizations) is based on the three layered hierarchical structure decomposition [2]. The top and intermediate layers are composed of cognitive, homogeneous, semi-autonomous agents, while the bottom layer contains cognitive, heterogeneous, semi-autonomous agents. The number of agents per layer depends on the layer's hierarchical level:

(i) the top level layer has exactly one agent;

(ii) the intermediate layer number of agents is problem-domain dependent - there can be further in layer hierarchical decomposition, both horizontal and vertical, according to the complexity of the domain being mapped;

(iii) the bottom layer number of agents is also problem-domain dependent - there is no hierarchical decomposition, and the amount of agents varies horizontally, based on the needs imposed by the problem-domain.

Time-sharing, agent performance, resources similarity and process nature are some of the criteria used for establishing the composition of the intermediate and bottom layers.

The required capabilities of the agents of the top and intermediate layers are planning, action coordination, communication and learning. These agents are called administrator-agents. The required capabilities of the agents of the bottom layer are task execution (domain expertise),

${ }^{1}$ Perception, memory and thinking . 
communications and learning. These agents are called executor-agents. From the cover-agent point of view, the intermediate and bottom set of inner agents are distributed and centralized.

There are two different sets of knowledge present in an organization: the corporate knowledge (CK) or the cover-agent knowledge, and the individual knowledge (IK) or the internal agent's .knowledge. The CK and IK are acquired, transformed and learnt by the inner agents during their interactions. The CK can be divided in two classes [3]. The first knowledge class, known as self model (SM), represents the styles, missions, plans, resources, processes, etc., of the inner world. The second knowledge class, denominated acquaintance model (AM) represents the outer or external world. The AM is divided in two sub-models: (i) the coveragents sub-model (CS) containing a local representation of the remaining cover-agents, and (ii) the society sub-model (SS) containing a local representation of the society. The continuous analysis of the agents' interactions plays an essential role: it acts as the knowledge acquisition interface that enables learning. Consequently, both sub-models ( CS and SS) are dynamic.

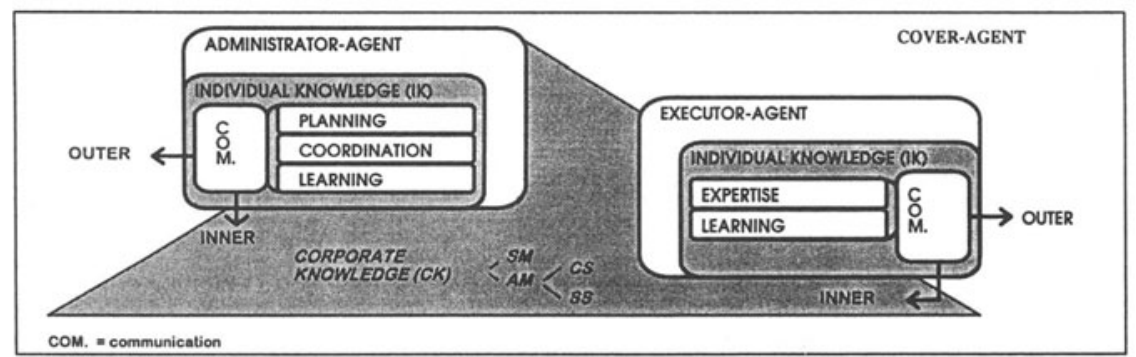

Figure 3 - A cover-agent.

The corporate knowledge is a shared knowledge base which is maintained and updated by the organization's inner agents. This corporate knowledge base contains (Figure 4) not only the organization's functional model but also encompasses the corresponding model of competencies. The functional model is supported by a general descriptor of the functions performed by the organization. The descriptor is an abstract representation of the functional objects and their relations, and provides an integrated model of the concepts, processes and resources of the organization. The acquisition, transformation and learning of new corporate knowledge performed during the execution of the organization tasks is oriented by this abstract descriptor of the corporate knowledge base.

The model of competencies defines the duties and the rights of each inner agent. The competencies of an inner agent can be viewed as a projection over the corporate knowledge base descriptor. The inner agent is responsible for maintaining, updating and learning about the concepts of the corresponding corporate knowledge subset defined by the adequate projection.

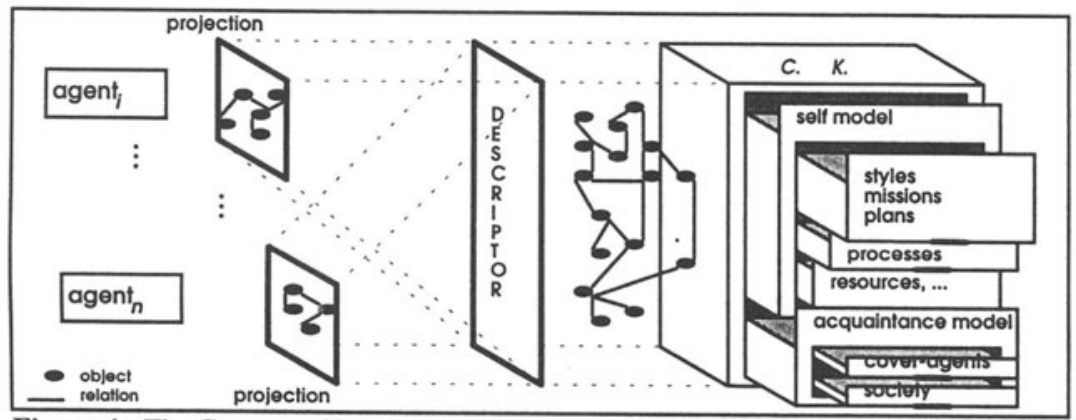

Figure 4 - The Corporate Knowledge. 


\section{THE PARTNER COVER-AGENTS' SELECTION PROCESS}

Our work is focused on a well-defined type of interaction: the interactions that occur between cover-agents acting as producers and cover-agents acting as suppliers. The producer's intent is to find the most adequate supplier ${ }^{2}$ of a good or service. A cooperative action is established when a supplier is contracted by a producer. This contracting is the result of a selection process. The selection process consists in sending an invitation (posted in the stand-news by the producer) to the society, followed by the evaluation of the received offers. Negotiation is invoked when conflicts arise. The contract will be celebrated with the supplier that satisfies the producer's constraints. In the specific setting of our approach we assume that the selection process is undertaken by the bottom layer executor-agents of the involved cover-agents. The cover-agents engaged in a cooperative mission have well-defined roles [3]: (i) the producers, or organizers are the cover-agents which are searching for cooperative partners, and (ii) the suppliers, or respondents, are the cover-agents that wish to participate in the proposed cooperative activity.

The evaluation process for each good or service to be contracted is based on the analysis of the existing local constraint list (CL). A CL is a conjunction of units denominated evaluation constraints (EC). They are used by the organizer to validate the respondents' proposals according to three criteria: (i) an organizational criterion that validates organizational attributes (structural, financial , etc.), (ii) a technical criterion that validates the characteristics of the good or service (color, dimension, etc.) and (iii) a commercial criterion that validates commercial attributes (price, quantity, etc.).

Each EC is a 5-tuple where:

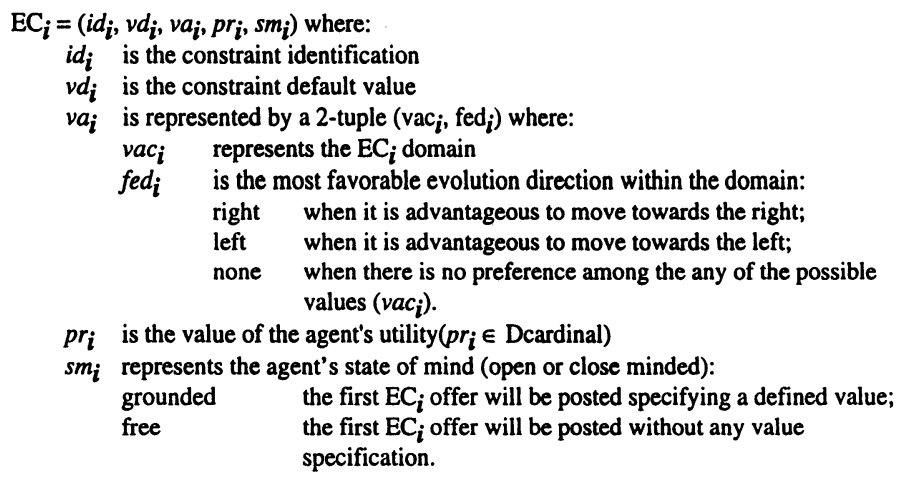

This sort of $\mathrm{CL}$ is called default_CL. As an example, is presented a default_CL, involving three constraints: color, price and payment_period.

\{ (color, blue, ((black, green, blue), none), 2, ground), (price, 30, ([0,33], left), 5, free),

(payment_period, 60, $([30,60,90]$, right), 2, ground) $]$.

Alternative CLs can be provided representing, either alternative constraint values or predefined constraint value's relaxation, which define the alternative bids. These new CLs are called alternative_CLs. As an example, is presented an alternative_CL, involving the same three constraints: color, price and payment_period.

I (color, blue, ((black, green, blue), none), 2, ground),

2 The most adequate supplier is the one that satisfies the constraints imposed by the producers. 
(price, 34, ([34, 36], left), 4, ground),

(payment_period, 120, ([120,150], right), 3, ground) ].

The union of the cartesian product of the domains of the ECs that belong to the default_CL with the cartesian product of the domains of the ECs that are specified in the alternative_CLs, define the plausible negotiation space state for a good or service. Every EC has two different utility parameters: (i) the first utility parameter denominated $\mathrm{pr}_{i}$ represents the EC's importance within the CL; and (ii) the second utility parameter expresses the most favorable evolution direction $\left(\mathrm{fed}_{i}\right)$ of the concept within its domain (left, right, none). The global utility of a CL is given by the sum of the individual ECs utility parameters ( $\mathrm{pr}_{i}$ and fed $\mathrm{d}_{i}$ ).

The respondents have, internally, the same CL representation data format. There is, however, one exception: the respondents do not communicate their states of mind. The selection process is started and ended by the organizers. It starts when the organizer sends the invitation to the standnews using the following data type:

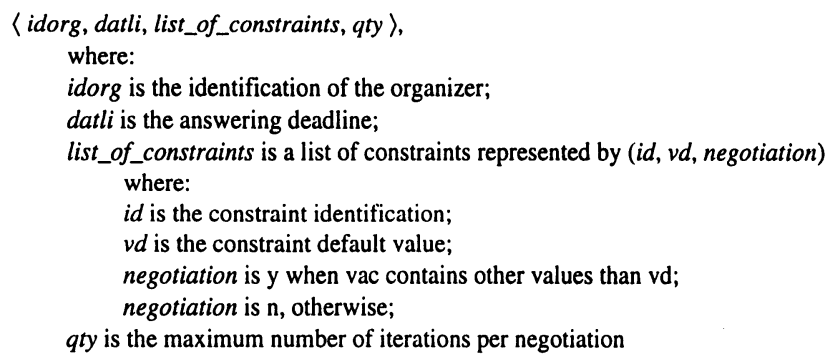

Example:

$\left\langle\text { agent }_{n}, 12,((\text { color, blue, y), (price, },, y),(\text { payment_period, } 60, y)), 10\right\rangle^{\prime}$

In our setting, the economic principles that guide the behavior of the organizations make them eager to cooperate.

Every cover-agent that has available resources continuously, poles the stand-news in search of new business opportunities. The invitations posted in the stand-news are analyzed by every one of these potential respondents. Those who have the necessary resources send their first offer to the organizer.

\subsection{The Evaluation Process}

The evaluation process is exclusively performed by the organizers. The goal of this process is to measure the distance between the specified demand and the received offers. In order to calculate these distances' values two filters are applied: a) Filter 1 is applied to the ECs that contain only default values, b) Filter 2 is applied to the remaining types of ECs. It computes the distance between the default and answered values for each remaining EC and for each respondent (dist $=$ default_value - answered_value).

Three situations may arise:

(i) there is only one respondent such that dist $\leq 0$ (this means that either the respondent offer satisfies (dist $=0$ ) the CL specified by the organizer, or that it may contain more attractive values (dist $<0$ ). Negative distances (dist $<0$ ) occur when the offered values are more advantageous than the proposed ones;

(ii) more than one respondent has presented attractive offers (dist $\leq 0$ ); 
(iii) there are no respondents that, totally or partially, fit into the initially specified default values (dist $>0$ ) for any or all ECs.

\subsection{The Negotiation Process}

The negotiation process is a decision making process, which helps the agents to satisfy their goals. The organizers look for respondents such that, $\forall \mathrm{EC}$, dist $\rightarrow \mathrm{Z},(\mathrm{Z} \leq 0)$. The respondents' aim, while trying to satisfy the organizer's $C L$, is to be contracted. In their effort to satisfy the organizer bids they make use of the available EC utilities. Organizers use the conflict resolution further negotiation. Conflicts occur when the distance calculated in the evaluation phase is either negative or positive, giving rise to the positive and negative conflict types [3].

The selection process has diverse halting conditions: (i) Through the celebration of a contract when only one respondent has met the bid's requisites, (ii) After a pre-defined number of evaluation-negotiation interactions. This value is defined by the organizer. This condition is met when: (a) more than one respondent has made tempting offers (dist $\leq 0$ ), (b) no agent is making offers, and (c) none of the respondents' offers are sufficiently close to what the organizer has specified (dist $>0$ ).

Before beginning the negotiation phase each involved agent selects one strategy (Figure 5), among those available in its strategies set. This choice is made based on the consultation of the following agent's knowledge: the self model (SM) and the acquaintance model (AM). In the case of the self model, the agent's style is what is more relevant. The agent's style is a metaknowledge that characterizes the agent inner and outer behavior. This style may be classified as win/win or win/lose. A win/win agent governs its activities with reciprocal advantage concepts towards its partners. It is characterized for taking into account, totally or partially, the answered values. A win/lose agent directs its activities in an egocentric fashion, solely moved by his own advantage. It is characterized for disregarding the answered values. The acquaintance model helps the agent to select its strategy based on what it knows about the involved cover-agent (CS) as well as about the society (SS) as a whole. At startup, ARTOR's cover-agents have empty acquaintance models. As a result of the established interactions, the AMs are, incrementally, built and refined.

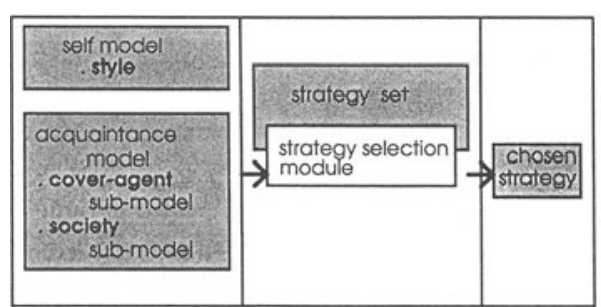

Figure 5 - Strategy choice.

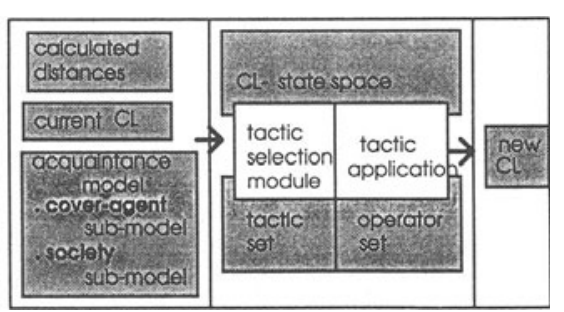

Figure 6 - Counter-proposal generation.

Once chosen the strategy, organizer and respondent, have to select the most adequate tactics. Each strategy has an associated number of tactics and operators that are applied to the current $\mathrm{CL}$ in order to create the new CL (Figure 6). The organizer selects the most appropriate tactics according to: (i) the current CL (ECs values); (ii) the calculated distances - when the offer exceeds the values of the previous demand (dist $\leq 0)$, the organizer employs the tactics that maximize its benefits. Otherwise it applies tactics that provide alternative_CLs and (iii) acquaintance model.

The respondent chooses the tactics that try to meet the organizers demand, based on the current CL and acquaintance model. 
Each iteration (Figure 7) is composed of an evaluation of the proposals $\left(\mathrm{CL}_{n}\right)$, followed by the generation of a counter-proposal $\left(\mathrm{CL}_{n+1}\right)$ and the subsequent sending of the new counterproposal $\left(\mathrm{CL}_{n+1}\right)$ to the involved agent(s). The generated values or counter-proposal (e.g., $\mathrm{CL}_{n+1}$ ) is the result of the application of the strategy, tactic and operator chosen.

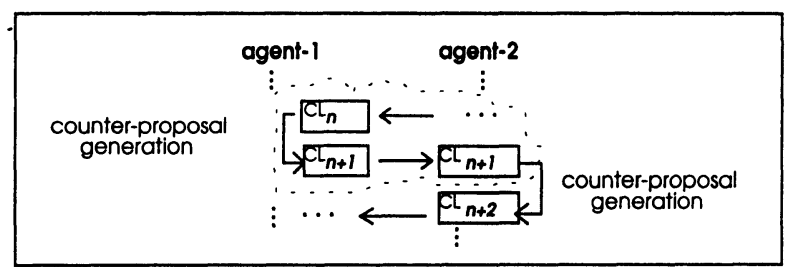

Figure 7 - An interaction.

The set of styles, the strategies, the tactics and the operators of an agent are represented as frames (Figure 8). Each style has an associated set of concept frames defining it. The set of applicable strategies is defined by the style. Associated to each strategy there are frames representing tactics which support the strategy enforcement via specific operators. Operators can increase, decrease or maintain the EC values.

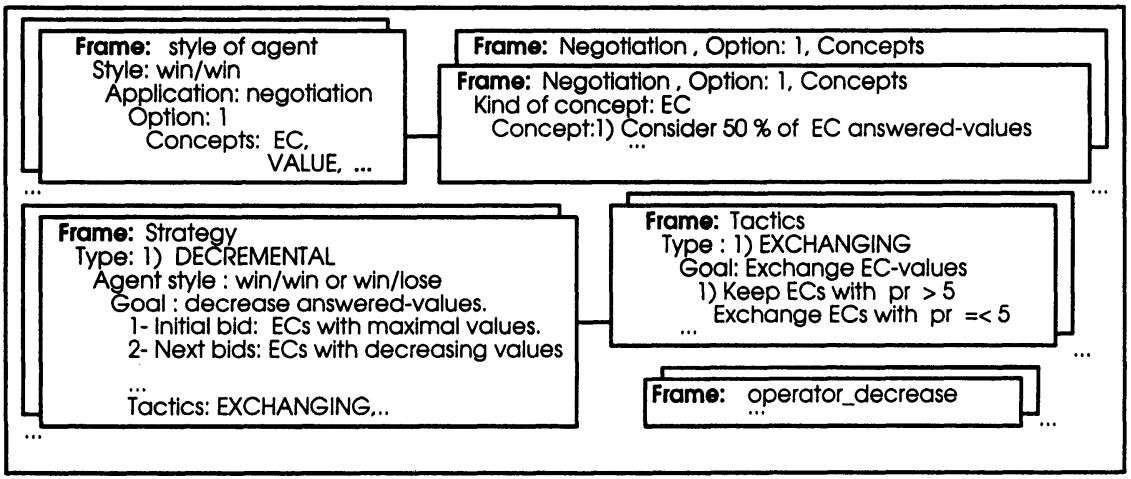

Figure 8 - Knowledge representation.

\subsection{Reasoning about Interactions}

The model chosen for the study of the discussed segment of the goods and services market is being implemented according to the DAI-MAS methodologies [4]. In this context, it is possible to detect a number of learning opportunities, based on the analysis of the interactions established among the cover-agents (organizations). In particular, ARTOR's cover-agents are focused on two specific learning opportunities:

(i) learning organizational concepts - The conceptualization of the behavior of other coveragents or the construction and refinement of the cover-agents sub-model (CS);

(ii) learning social concepts - The conceptualization of the behavior of the society, as a whole, or simply, the construction and refinement of the society sub-model (SS).

In the ARTOR's model, the learning capabilities lay on the individual inner agents. The learning capability of a cover-agent, as a whole, is the sum of the individual inner agents' capabilities. The inter-agent exchanges that occur during the evaluation and negotiation steps, constitute the set of examples used for learning. Each example is a triplet $\left(\mathrm{P}_{r l}, \mathrm{CL}_{n+1}, \mathrm{CL}_{n+2}\right)$ (Figure 9). $\mathrm{P}_{r l}$ is the internal transformation process used to generate the new constraint list from the current $\mathrm{CL}\left(\mathrm{CL}_{n}\right)$. The $\mathrm{CL}_{n+1}$ is the new internal constraint list that will be generated 
once $\mathrm{P}_{r 1}$ is applied to $\mathrm{CL}_{n}$. The $\mathrm{CL}_{n+2}$ is the constraint list that will be externally produced upon the reception of $\mathrm{CL}_{n+1}$. The learning will be concentrated on the virtual consequence dependency that can be established between the internally generated $\mathrm{CL}_{n+1}$ and the externally generated $\mathrm{CL}_{n+2}$.

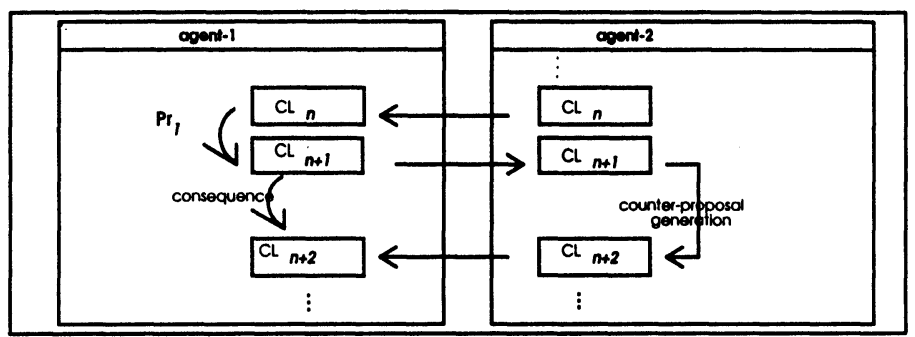

Figure 9 - An inter-agent exchange.

The purpose of the individual learning activity (within different layers- top, intermediate and low) is to hypothesize assumptions regarding the behavior of others. This type of learning is based on the analysis of the CLs changes that exist during the selection process. From the comparative analysis of consecutive CLs $\left(\mathrm{CL}_{n+1}\right.$ and $\left.\mathrm{CL}_{n+2}\right)$ a classification of the experienced EC changes emerges. The calculated deltas, or variations are cataloged in three possible classes:

(i) null delta or = class - when the ECs have remained unchanged;

(ii) positive delta or $\uparrow$ class - when the ECs have experienced an incremental alteration;

(iii) negative delta or $\downarrow$ class - when the ECs have suffered a decremental variation.

The calculated deltas represent the difference between the desired and offered EC values. In (Figure 10) a default_CL which contains three constraints (EC) denoted by a, b, c is presented. The default ECs values, expected by the organizer, are in the central line of the first column $\left(\mathrm{CL}_{i}\right)$. The other columns represent posterior states which were generated by respondent and organizer during the selection process.

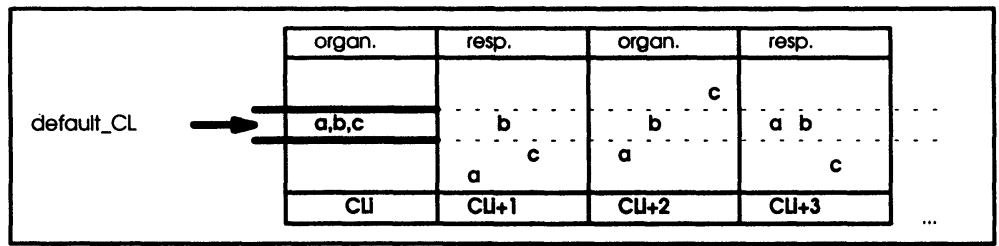

Figure 10 - An example.

In this case the calculated deltas are:

\begin{tabular}{|l|l|l|l|l|l|l|l|}
\hline $\mathrm{CL}$ & a & b & c & & a & b & c \\
\hline $\mathrm{CL}_{i}$ & initial & initial & initial & $\mathrm{CL}_{i+1}$ & $\downarrow,[$ value] & $=$ & $\downarrow,[$ value] \\
\hline $\mathrm{CL}_{i+2}$ & $\uparrow$, , [value] & $=$ & $\uparrow,[$ value] & $\mathrm{CL}_{i+3}$ & $\uparrow,[$ value] & $=$ & $\downarrow,[$ value] \\
$\Rightarrow \quad$ organizer
\end{tabular}
$\Rightarrow \Rightarrow \quad \Leftarrow$

The learning process has two phases: (i) example analysis and (ii) submission of the new example set to the learning algorithms The analysis of the example consists in establishing for each constraint (EC) the extremes of the negotiated values. The applied algorithm uses both inductive and deductive reasoning. The agents involved in a selection process (the organizer and the respondent agents) will have, by the time it finishes, a more accurate internal model of its 
counterpart. The quality of the model created depends on the quantity of information made available during the selection process, or in other words, the richness of the set of examples provided. The inductive and deductive learning methodologies are applied over the same set of examples but distinct complementary concepts are obtained. Through the application of: (i) the inductive learning paradigm [5], higher level knowledge about the behavior of the CLs during the selection process is produced, (ii) the deductive learning algorithm based on (EBL, $\mathrm{mEBG}$ ) [6], higher level knowledge concerning the style of the agents involved in the selection process, is generated.

In the first case (i), the organizer and the respondent not only learn new organizational concepts concerning the evolution and relative importance ${ }^{3}$ of the negotiated constraints, but also learn new local social concepts regarding their respective social roles/behaviors. In the second case, the agents use as background knowledge (BK) the knowledge present in each agent style, strategies and tactics slots to deduce new local social perspectives. However, when the background knowledge does not support deductive reasoning, deductive learning is not possible. In such circumstance specialize concepts are hypothesized.

Generically, the learnt assumptions concerning the organizational concepts are represented in the cover agent sub-model (CS) by a 〈id_ag, assumptions $\rangle$ slot, while the social concepts are represented in the society sub-model (SS) using a 〈assumptions〉 slot, where id_ag is the identification of the counterpart agent, and assumptions are the learnt assumptions (rules, propositions, etc.).

As an example, and according to the interaction described in Figure 10, the organizer (agent 1 ) has learnt about the respondent (agent 2 ), that in those specific circumstances $\mathrm{EC}_{\mathbf{b}}$ remains constant:

< agent $\mathrm{EC}_{\mathbf{b}}=$ default_value when $\mathrm{EC}_{\mathrm{a}} \subset\left[\mathrm{a}_{1}, \mathrm{a}_{\mathbf{n}}\right]$ and $\mathrm{EC}_{\mathbf{c}} \in\left\{\mathrm{c}_{1}, \mathrm{c}_{\mathrm{i}}\right\}>$.

Assuming that after the selection process of executor agent 1 , a quality control process occurred at executor agent 11 , and that both executor agents are managed by the same administrator agent $i$ (intermediate layer agent), a higher level concept (Figure 11) about external agent 2 can be learnt:

Agent 1 < agent 2 , was selected to provide the specified goods/services >.

Agent $11<$ agent $_{2}$, ISO quality requirements $=92 \%>$.

Agent $_{i}<$ agent $_{2}$, satisfaction $=\mathrm{OK}>$.

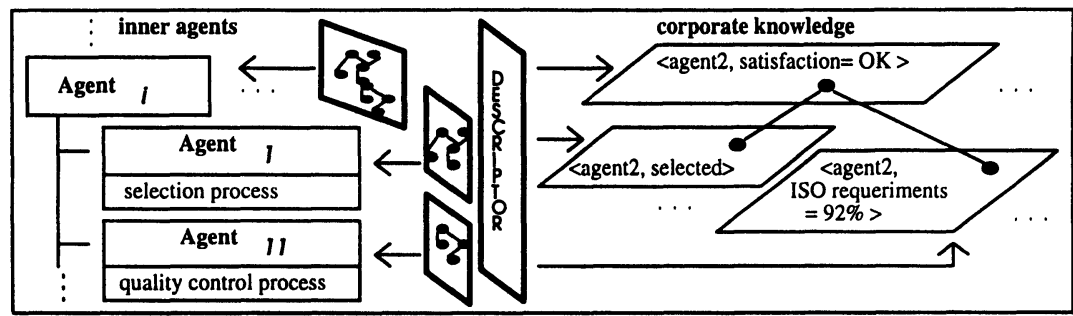

Figure 11 - Higher level concept.

In future selection and quality control processes involving this same good/service the coveragent of executor agents 1 and 11 , will take into account the satisfaction concept learnt about the cover-agent of executor agent 2 .

\footnotetext{
${ }^{3}$ The importance of a constraint can be measured in terms of its weight in the final sucess or failure of the selection process: hard constraints are non relaxable and soft contraints are relaxable.
} 


\section{CONCLUSIONS}

The work we have described in this paper, aims at contributing to the computer simulation of the interacting of organizations. Several different approaches for modeling of the goods and services market as well as its organizations have been attempted and implemented. The work of Wellman [7] and the research of Barbuceanu and Fox [8] are some good examples of the currently undergoing investigation on this subject. Conflict resolution via negotiation in multiagent systems is also based on previous work by Oliveira [9] and by Sycara [10], while cooperative learning has been explored in Sian [11] and others.

The contribution of our work lies, not only, on the model proposed for the society of organizations, but also, on the distributed learning methodology applied to the established partnerships, in particular, and to the community, in general. The combination of the three layered hierarchical structure with the in-layer multiagent architecture provides a rich and adequate setting for the representation of complex organizations. The inherent modularity of the presented architecture allows the representation of organizations with different levels of complexity, by means of social grouping. In particular, our efforts are concentrated on the analysis of a specific type of interaction which is one of the basic step to assemble a virtual organization: the cooperative interaction between producers and suppliers. The producer's intent is to find the most adequate supplier, according to a set of specified constraints. The supplier's aim is to be contracted while trying to meet every producer's demand. The mechanism used to select the most adequate partner is based on a selection process which is composed of evaluation, negotiation and conflict resolution phases. Once an interaction is established, every exchanged constraint list is collected. The set of collected constraint lists constitutes a cooperative example, which can be used by the learning algorithms to generate assumptions about the behavior of the involved organizations, as well as about the society as a whole.

\section{ACKNOWLEDGMENTS}

The first author's current research is being supported by PUC-Pr and CNPq - Conselho Nacional de Pesquisa, Brazil, Grant Number 200413/92-9.

\section{REFERENCES}

[1] Engelmore, R., Morgan, T. (1988) Blackboard Systems. Addison - Wesley Publishing Company.

[2] Simon, H.A., Decision Making and Organizational Design. In: Pugh, D.S.ed. Organizational Theory. Penguin Books. p.189-212.

[3] Oliveira, et.al. (1993) Negotiation and Conflict Resolution within a Community of Cooperative Agents. In: Proceedings of The First International Symposium on Autonomous Decentralized Systems, Kawasaki, Japan.

[4] Gasser, L. Huhns, M.N. (1989) Distributed Artificial Intelligence, vol.II, Pitman Publishing, London.

[5] Michalski, R.S. (1990) Learning Flexible Concepts: Fundamental Ideas and a Method Based on Two-Tired Representation. In: Machine Learning - An Artificial Intelligence Approach, vol. III, Edited by Yves Kodratoff and Ryszard Michalsky, Morgan Kaufmann Publishers, Inc.

[6] Kodratoff, Y. (1990) Learning Expert Knowledge by Improving the Explanations Provided by the System. In: Machine Learning - An Artificial Intelligence Approach, vol. III, Edited by Yves Kodratoff and Ryszard Michalsky, Morgan Kaufmann Publishers, Inc. 
[7] Wellman, M.P. (1993) A Market-Oriented Programming Environment and its Application to Distributed Multicommodity Flow Problems. In: Journal of Artificial Intelligence Research, 1 (1993) 1-23, AI Access Foundation and Morgan Kaufmann Publishers.

[8] Barbuceanu, M., Fox, M.S. (1994) The Information Agent: An Infrastructure for Collaboration in the Integrated Enterprise. In: Proceedings of the 2nd International Working Conference on Cooperating Knowledge Based Systems, Editor S.M.Deen, University of Keele.

[9] Oliveira, E., Mouta, F. (1993) Distributed AI Architecture Enabling Multi-Agent Cooperation. In: Industrial and Engineering Applications of Artificial Intelligence and Expert Systems, Edited by Paul W.H. Chung, Gillian Lovegrove and Moonis Ali, Gordon and Breach Science Publishers.

[10] Sycara, K.P. (1989) Multiagent Compromise via Negotiation. In: Distributed Artificial Intelligence, vol.II, Edited by Les Gasser and Michael N. Huhns, Pitman Publishing, London.

[11] Sian, S.S. (1991) Adaptation Based on Cooperative Learning in Multi-Agent Systems. In: Decentralize A.I. - 2, Edited by Yves Demazeau and Jean-Pierre Muller, Elsevier Science Publishers B.V. 